\title{
Optically Detected X-ray Absorption Spectroscopy Measurements as a Means of Monitoring Corrosion Layers on Copper
}

\author{
Mark G. Dowsett, ${ }^{*, t}$ Annemie Adriaens, ${ }^{\ddagger}$ Gareth K. C. Jones, ${ }^{\dagger}$ Nigel Poolton, ${ }^{\S}$ Steven Fiddy," and \\ Sergé Nikitenko ${ }^{\perp}$
}

Department of Physics, University of Warwick, Coventry CV4 7AL, U.K., Department of Analytical Chemistry, Ghent University, Krijgslaan 281 S12, B9000 Ghent, Belgium, Institute of Mathematical and Physical Sciences, University of Wales Aberystwyth, Ceredigion SY23 3BZ, Wales U.K., and Faculty of Applied Sciences, Delft University of

Technology, Mekelweg 15, 2629 JP Delft, The Netherlands, Science \& Technology Facilities Council, Daresbury

Laboratory, Daresbury Science \& Innovation Campus, Warrington, WA4 4AD, U.K., and Netherlands Organisation for

Scientific Research (NWO), DUBBLE@ESRF, BP 220, 38043 Grenoble, Cedex 9, France

XANES and EXAFS information is conventionally measured in transmission through the energy-dependent absorption of X-rays or by observing X-ray fluorescence, but secondary fluorescence processes, such as the emission of electrons and optical photons (e.g., 200-1000 nm), can also be used as a carrier of the XAS signatures, providing complementary information such as improved surface specificity. Where the near-visible photons have a shorter range in a material, the data will be more surface specific. Moreover, optical radiation may escape more readily than X-rays through liquid in an environmental cell. Here, we describe a first test of optically detected X-ray absorption spectroscopy (ODXAS) for monitoring electrochemical treatments on copper-based alloys, for example, heritage metals. Artificially made corrosion products deposited on a copper substrate were analyzed in air and in a $1 \%(\mathrm{w} / \mathrm{v})$ sodium sesquicarbonate solution to simulate typical conservation methods for copper-based objects recovered from marine environments. The measurements were made on stations 7.1 and 9.2 MF (SRS Daresbury, UK) using the mobile luminescence end station (MoLES), supplemented by XAS measurements taken on DUBBLE (BM26 A) at the ESRF. The ODXAS spectra usually contain fine structure similar to that of XAS spectra measured in X-ray fluorescence. Importantly, for the compounds examined, the ODXAS is significantly more surface specific, and $>98 \%$ characteristic of thin surface layers of $0.5-1.5-\mu \mathrm{m}$ thickness in cases where $X$-ray measurements are dominated by the substrate. However, EXAFS and XANES from broadband optical measurements are superimposed on a high background due to other optical emission modes. This produces statistical fluctuations up to double what would be expected from normal counting statistics because the data retain the absolute statistical fluctuation

\footnotetext{
* To whom correspondence should be addressed. E-mail: m.g.dowsett@ warwick.ac.uk.

${ }^{\dagger}$ University of Warwick.

* Ghent University.

$\S$ University of Wales Aberystwyth, and Delft University of Technology.

" Daresbury Science \& Innovation Campus.

${ }^{\perp} \mathrm{ESRF}$.
}

in the original raw count, while losing up to $70 \%$ of their magnitude when background is removed. The problem may be solved in future through optical filtering to isolate the information-containing band, combined with the use of higher input X-ray fluxes available on third-generation light sources.

Electrochemical techniques provide a huge potential range of treatments for cultural heritage and other metals. These include the removal and passivation of corrosion layers, the application of protective coatings, and in situ monitoring during storage and stabilization. However, in the area of heritage science, because of the irreplaceable nature of many historical artifacts, each process needs to be thoroughly tested and understood, ideally before first use. Elsewhere we describe an electrochemical cell (eCell) that allows real metal surfaces (rough, heterogeneous) to be studied using X-ray diffraction (XRD) and X-ray absorption spectroscopy (XAS) in synchrotron beam lines so that coincident time-resolved electrochemical and X-ray data can be gathered (socalled spectroelectrochemistry). ${ }^{1}$ Structural information from the $\mathrm{X}$-ray technique is then used to identify reaction products and correlate their appearance/disappearance and rates of change of intensity with features in the electrochemical monitoring channel (e.g., corrosion potential measured as the open circuit potential (OCP) of the cell, voltammetry, and electrochemical impedance spectroscopy (EIS)). So far, the eCell has been successfully deployed using XRD and XAS combined with OCP and EIS. Experiments have involved the in situ monitoring of the surface and electrochemical behavior of archeological copper alloys and lead. ${ }^{2-6}$

(1) Dowsett, M.; Adriaens, A. Anal. Chem. 2006, 78 (10), 3360-3365.

(2) Leyssens, K.; Adriaens, A.; Dowsett, M.; Schotte, B.; Oloff, I.; Pantos, E.; Bell, A.; Thompson, S. Electrochem. Commun. 2005, 7, 1265-1270.

(3) Adriaens, A.; Dowsett, D.; Leyssens, L.; Van Gasse, B. Anal. Bioanal. Chem 2007, 387 (3), 861-868.

(4) Adriaens, A.; Dowsett, M. Surf. Eng. In press; doi: 10.1179/174329408 × 281759.

(5) Adriaens, A.; Dowsett, M.; Jones, G. K. C.; Leyssens, K.; Nikitenko, S. An in-situ X-ray absorption spectroelectrochemistry study of the response of artificial chloride corrosion layers on copper to remedial treatment. J. Anal. Atom. Spectrosc. In press

Analytical Chemistry, Vol. 80, No. 22, November 15, 2008

8717

Published on Web 10/15/2008 
Optically detected X-ray absorption spectroscopy (ODXAS) has the potential to improve on the lack of surface specificity of X-ray diffraction and absorption measurements using beams with energies of $>8 \mathrm{keV}$ where the penetration and escape depths exceed the thickness of the layers of interest. It is well-known that the escape depths of optical photons and electrons produced by Auger de-excitation and other processes occurring as a consequence of the X-ray photoionization event are in the range of hundreds of nanometers to $<5 \mathrm{~nm}$ (unless the material is transparent to the photons), so that the detection of these, rather than the X-ray fluorescence yield, has the potential to enhance the surface specificity. Although for measurements on surfaces immersed in an electrochemical cell it might in principle be possible to detect the electron current injected into the electrolyte, approximate calculations show that the currents involved are within the expected random noise level on the electrochemical currents, so photon detection is the better option. Moreover, for cases where a significant amount of a surface layer dissolves in the electrolyte, we also observe strong effects due to selfabsorption when detecting X-ray fluorescence. These lead to emission that is dominated by the electrolyte chemistry rather than the surface chemistry. Different absorption processes will affect optical photons emitted from the immersed surface, leading to complementary modes of analysis.

ODXAS is an emerging field of X-ray absorption spectroscopy, which has been applied, for example, to the characterization of various types of oxides, ${ }^{7-10}$ and is giving rise to new generations of microscopy tools. ${ }^{11}$ The equipment can be simple: a photomultiplier tube or broadband CCD detector is used in place of an X-ray detector for the observation of X-ray excited optical luminescence. ${ }^{12-15}$ Since optical photons can only travel short distances within an opaque sample, the resulting spectra are characteristic of a layer less than $200 \mathrm{~nm}$ thick on the sample surface. This level of surface specificity is ideal for the observation of chemical reactions occurring on an electrode in an electrochemical cell.

Here, we report the results from spectra taken from reference powders and corrosion layers relevant to archeological copper artifacts, viz.: cuprite (copper(I) oxide), nantokite (copper(I) chloride), and atacamite and paratacamite (isomeric copper hydroxychlorides). Corrosion layers were also measured while immersed in $1 \% \mathrm{w} / \mathrm{v}$ sodium sesquicarbonate solution, simulating a common conservation treatment. A further objective was to acquire spectra as quickly as possible to achieve the (minimum) time resolution (20-30 min), which is useful when studying reactions in the eCell. Cuprite $\left(\mathrm{Cu}_{2} \mathrm{O}\right)$ is regularly found on ancient and modern copper artifacts and is a stable corrosion product. ${ }^{16}$

(6) Dowsett, M.; Adriaens, A.; Schotte, B.; Jones, G. K. C.; Bouchenoire, L. Real time spectroelectrochemical study of the growth process of a lead decanoate coating as corrosion inhibitor for lead surfaces. Surf. Interf. Anal., submitted.

(7) Nakamura, T.; Takahashi, T.; Poolton, N. R. J.; Malins, A. E. R. Appl. Surf. Sci. 2005, 244, 318-321.

(8) Poolton, N. R. J.; Pantos, E.; Hamilton, B.; Denby, P. M.; Johnsen, O. Phys. Status Solidi B 2004, 241, 3656-3633.

(9) Larcheri, S.; Rocca, F.; Jandard, F.; Pailharey, D.; Graziola, R.; Kuzmin, A.; Purans, J. Rev. Sci. Instrum. 2008, 79, 013702-1-013702-9.

(10) Ko, J. Y. P.; Heigl, F.; Yiu, Y. M.; Zhou, X. T.; Regier, T.; Blyth, R. I. R.; Sham, T. K. Can. J. Chem. 2007, 85 (10), 853-858.

(11) Armelaont, L.; Heigl, F.; Jurgensen, A.; Blyth, R. I. R.; Regier, T.; Zhou, X. T.; Sham, K. T. J. Phys. Chem. 2007, 111 (28), 10194-10200.
Among the copper chlorides, nantokite $(\mathrm{CuCl})$, atacamite $\left(\mathrm{Cu}_{2}(\mathrm{OH})_{3} \mathrm{Cl}\right)$, and a mixture of atacamite and paratacamite (both isomers of $\mathrm{Cu}_{2}(\mathrm{OH})_{3} \mathrm{Cl}$ ) were selected. Nantokite is considered as the main catalytic agent for active corrosion. Its presence on the surface of a metal can create long-term problems with regard to the stability of an object. In fact, bronze disease or pitting corrosion is usually attributed to nantokite. ${ }^{16}$ Atacamite and paratacamite are two other important chlorides in copper alloy corrosion. They are often considered as end products and are formed on top of the active corrosion areas. Atacamite is the most common of the $\mathrm{Cu}_{2}(\mathrm{OH})_{3} \mathrm{Cl}$ isomers but often changes to paratacamite. ${ }^{16}$

In the rest of the paper, "XAS" refers to measurements of the transmitted or fluorescent X-ray flux, and ODXAS refers to measurement of wavelengths from near-IR to near-UV. The ODXAS spectra are compared to conventional XAS spectra under different circumstances (dry and in solution).

\section{EXPERIMENTAL SECTION}

Sample Preparation. Powder samples of $\mathrm{Cu}_{2} \mathrm{O}$ (for comparison with cuprite, Fluka, >99\%) and $\mathrm{Cu}$ (I) Cl (for comparison with nantokite, Fluka, >97\%) were purchased. Atacamite and paratacamite powders are not commercially available and were therefore synthesized using methods from two different sources. First, atacamite was prepared by adding a $50 \mathrm{~mL}$ of $0.1 \mathrm{M} \mathrm{Na}_{2} \mathrm{CO}_{3}$ (Fluka, > 99.5\%) solution dropwise to a stirred solution of 100 $\mathrm{mL}$ of $0.1 \mathrm{M} \mathrm{CuCl}_{2} \cdot 2 \mathrm{H}_{2} \mathrm{O}$ (Aldrich, $>99 \%$ ) boiling at reflux, ${ }^{17}$ while paratacamite was prepared by boiling $1 \mathrm{~g}$ of $\mathrm{CuO}$ (Fluka, >99\%) in $500 \mathrm{~mL}$ of an aqueous solution containing $150 \mathrm{~g}$ of $\mathrm{CuCl}_{2} \cdot 2 \mathrm{H}_{2} \mathrm{O}$ (Aldrich, >99\%). ${ }^{17}$ In both cases, the slurry was filtered, washed, and dried at $333 \mathrm{~K}$ after $5 \mathrm{~h}$. The samples were crushed to a very fine powder, mixed with a light silicone-free grease (Apiezon M, M \& I Materials Ltd.), and deposited on a clean polycrystalline diamond surface for analysis. In addition, both materials were made according to the methods described by Pollard: ${ }^{18}$ Paratacamite was prepared by dripping $9 \mathrm{~mL}$ of $0.1 \mathrm{M} \mathrm{NaOH}$ solution into $6 \mathrm{~mL}$ of $0.1 \mathrm{M} \mathrm{CuCl}_{2}$ while stirring. The solution was then filtered immediately to collect the product. Atacamite was prepared using $13 \mathrm{~mL}$ of $0.05 \mathrm{M} \mathrm{NaOH}$ solution dripped and stirred into 1.0 $\mathrm{L}$ of a solution made from $0.00425 \mathrm{M} \mathrm{CuCl}_{2}$ and $0.5 \mathrm{M} \mathrm{NaCl}$. This was filtered to collect the product after standing for $20 \mathrm{~h}$.

Simulated corrosion layers were made using protocols described below on circular copper coupons, $12 \mathrm{~mm}$ in diameter (Advent, purity 99.9\%), with all but the front surface encapsulated in acrylic resin. These were made in the form of electrodes for the eCell. ${ }^{1}$ They were prepared by grinding on P 1200-grit SiC paper to obtain a fresh surface, followed by polishing to a mirror finish as judged by eye (typical both of much museum and also new metalwork) using a cloth covered with $1 \mu \mathrm{m}$ alumina paste made by mixing the powder with deionized water. Any adherent $\mathrm{Al}_{2} \mathrm{O}_{3}$ particles on the surface were removed by immersing the samples in a propan-2-ol ultrasonic bath for 15 min and rinsing them thoroughly with propan-2-ol.

The coupons were corroded artificially as follows: ${ }^{17}$ To obtain a cuprite layer, the copper samples were polarized anodically at $320 \mathrm{mV}$ (vs a normal hydrogen electrode) for $16 \mathrm{~h}$ in a $0.1 \mathrm{M}$ $\mathrm{Na}_{2} \mathrm{SO}_{4}$ solution (Fluka, >99\%). Copper covered with nantokite was obtained by immersing pure coupons for $1 \mathrm{~h}$ in a saturated $\mathrm{CuCl}_{2} \cdot 2 \mathrm{H}_{2} \mathrm{O}$ solution (Aldrich, $>99 \%$ ). After rinsing with deionized 
water, they were exposed to the air overnight. For the atacamite, a solution of $15.07 \mathrm{~g}$ of ammonium carbonate (Sigma-Aldrich, ammonia $>30 \%$ ) and $10.02 \mathrm{~g}$ of ammonium chloride (SigmaAldrich, $99.998 \%$ ) in $100 \mathrm{~mL}$ of deionized water was prepared. One drop of the solution was used to wet the surface twice a day for 5 days. Between each wetting, the samples were left to dry in air. After the period of five days, the samples were left in the air for a further five days. The protocol used to obtain a mixture of atacamite and paratacamite differed only from the protocol used to obtain atacamite in the type of solution used: a solution of 10.02 $\mathrm{g}$ of $\mathrm{Cu}\left(\mathrm{NO}_{3}\right)_{2} \cdot 3 \mathrm{H}_{2} \mathrm{O}$ (VWR International, $98-103 \%$ ) and $10.01 \mathrm{~g}$ of $\mathrm{NaCl}$ (Fluka, >99.5\%) in $100 \mathrm{~mL}$ of deionized water was used.

The average thicknesses of the layers so produced are $\sim 0.6-1.5 \mu \mathrm{m}$ for the cuprite and nantokite, and hundreds of micrometers for the atacamite and atacamite/paratacamite mixture. The cuprite and nantokite measurements were obtained by ion-beam sputtering through the layer using a $500 \mathrm{eV}$ oxygen beam, stopping at the copper surface, and measuring the thickness removed using a DEKTAK 3030 surface profilometer (Sloan Corp. Inc.). The atacamite and atacamite/paratacamite layer thicknesses are visual estimates and the surface roughness is of the same order as the thickness-typical of a corroded surface.

For the immersion experiments, we needed a $1 \%(\mathrm{w} / \mathrm{v})$ sodium sesquicarbonate solution. The latter was prepared by dissolving $11.89 \mathrm{~g} / \mathrm{L}$ of $\mathrm{Na}_{2} \mathrm{CO}_{3} \cdot \mathrm{NaHCO}_{3} \cdot 2 \mathrm{H}_{2} \mathrm{O}$ (Sigma-Aldrich) in deionized water ( $\mathrm{pH} \mathrm{10)}$.

\section{ELECTROCHEMICAL CELL}

The prototype and later versions of eCell as used here (Figure 1A) are described in more detail elsewhere. ${ }^{1,3}$ For the present purpose, a light-tight cap made from black Delrin, which supports the optical system used for ODXAS, has been added (Figure 1B). The cap admits X-rays to the cell through a slit. The cell window used in these experiments was optically transparent "cooking film" made from $10-\mu \mathrm{m}$-thick low-density polyethylene (LDPE). For comparison, the mean transmission of 60- $\mu \mathrm{m} \mathrm{LDPE}$ with a low impurity level is $>93 \%$ and decreases by less than $4 \%$ across the wavelength range $300 \mathrm{~nm}-1 \mu \mathrm{m} .{ }^{19}$ This seals easily to the top of the cell, and since the windows adsorb chlorides (for example) from the cell electrolyte during a reduction process, the fact that it is inexpensive and disposable was considered important. During spectroelectrochemical analysis, the surface of interest (the working electrode in the cell) is positioned at $\sim 125 \mu \mathrm{m}$ from the inside of the window, thus remaining in contact with a layer of electrolyte. The preliminary ODXAS tests described below were conducted using or simulating this configuration.

ODXAS Analyses. ODXAS experiments were performed at stations 7.1 and 9.2 MF of the SRS (Daresbury). Components from the Mobile Luminescence End Station (MoLES) were used to acquire the $\mathrm{Cu}$ K-edge $(8.979 \mathrm{keV})$ ODXAS spectra. ${ }^{12,20}$

The setup is schematically shown in Figure 1B. The X-rays enter the cell at $45^{\circ}$ to the electrode surface (located toward the top of the cell), through the slit in the cell cap. The detection system has its axis normal to the sample surface and consisted of a UV-grade low-fluorescence fused-silica lens with a focal length of $30 \mathrm{~mm}$ (with the sample in the first focal plane) followed by a condenser lens with a 50-mm focal length and a UV-green sensitive bialkali photomultiplier tube with a 22-mm-diameter photocathode. A simple optical kit from MoLES allowed wavelength-specific
(A)
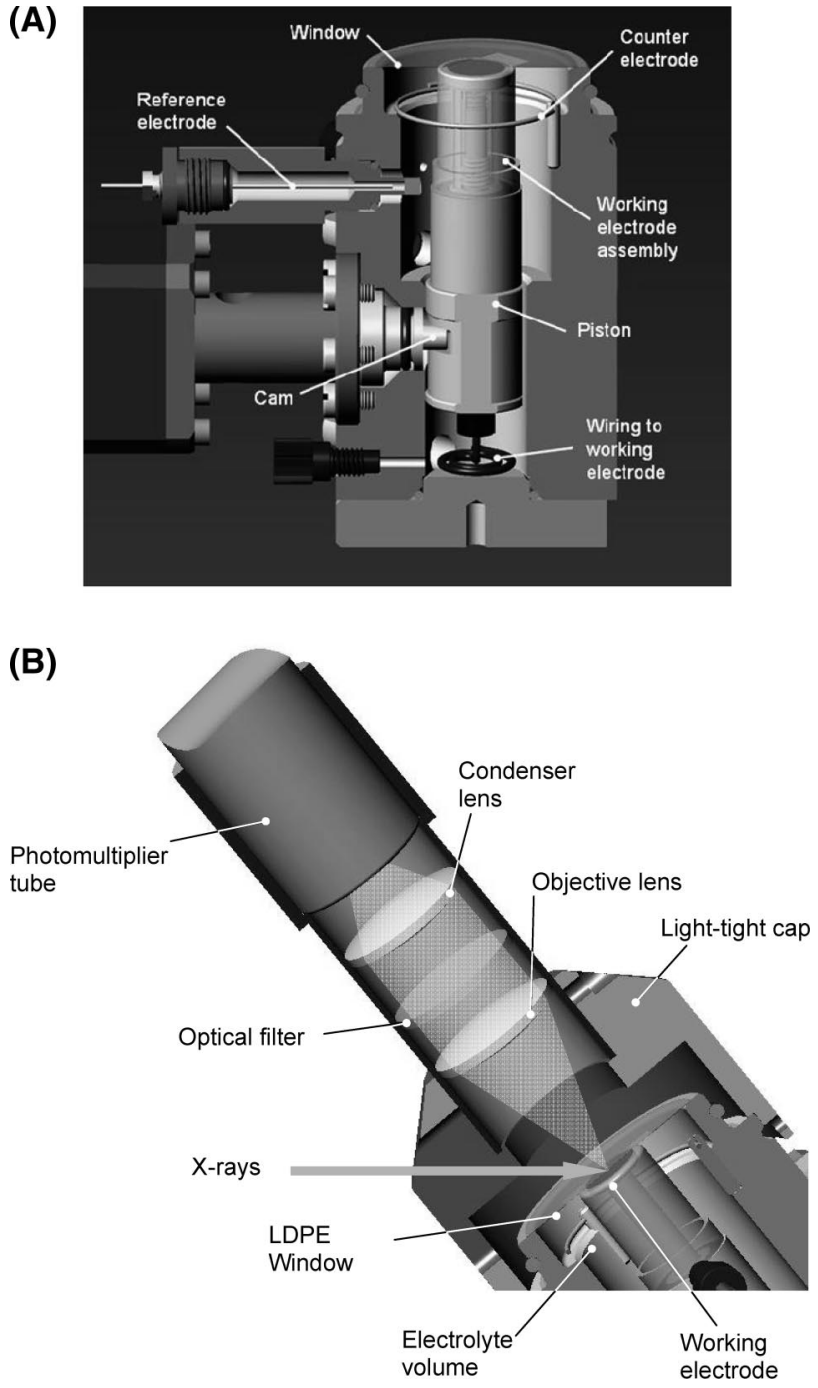

Figure 1. (A) Scheme of electrochemical cell. (B) Cutaway diagram of the optical system mounted on the electrochemical cell.

band-pass filters to be placed in the parallel beam between the lenses. The X-ray energy range used was $8.73 \mathrm{keV} \leq E \leq 9.53$ $\mathrm{keV}$. The beam footprint on the electrode surface was measured with light-sensitive paper to be $\sim 8 \mathrm{~mm} \times 1 \mathrm{~mm}$, although the light optical system will vignette the emission from toward the ends of the line.

The entire experiment was wrapped in aluminum foil and black cloth, and the station hutch operated in darkness to suppress background signal as much as possible. Total background due to dark counts and stray light could be kept at $\sim 100$ counts/s with care.

XAS Analyses. XAS experiments were performed at DUBBLE (Station BM26A, European Synchrotron Radiation Facility, Grenoble, France). $\mathrm{Cu}$ K-edge spectra were recorded as a function of energy over the range 8.9-9.6 keV. Measurements were performed either in transmission (powders) or in fluorescence mode for powders and electrodes measured dry or immersed in the eCell. The scan time was $60 \mathrm{~min}$ for the powders and $20 \mathrm{~min}$ for the other samples. The latter is a compromise between obtaining adequate statistics in the EXAFS region and sufficient time resolution to follow the solution chemistry. Two Oxford Instruments ion chambers were 


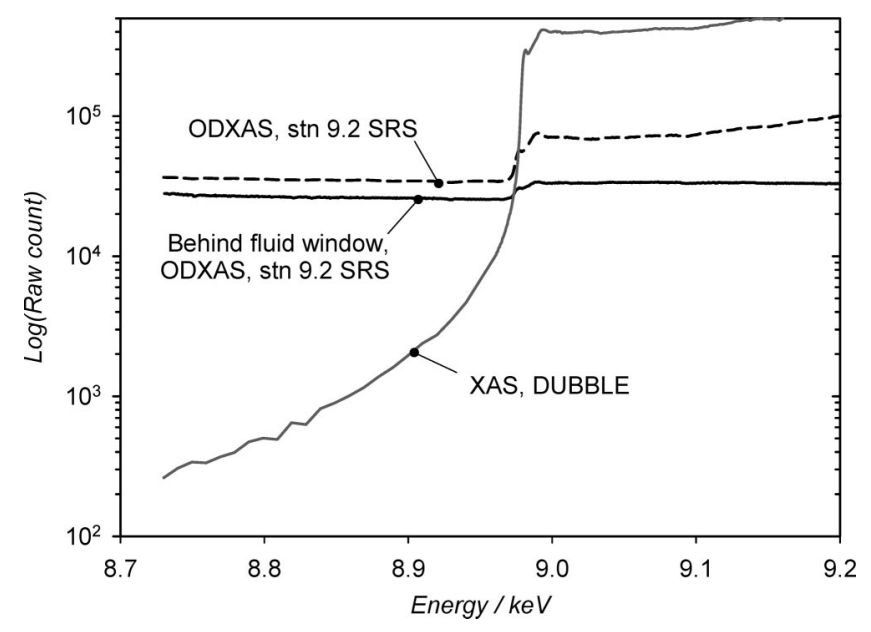

Figure 2. Logarithmic plot of raw counts in XAS and ODXAS of cuprite obtained at the different stations used. Note the high background on the ODXAS measurements due to optical processes other than those carrying the XAS modulation. (In this case, the background is that part of the spectrum recorded at energies of $<8.5$ keV.)

used for transmission experiments. Fluorescence measurements were made using the X-ray beam at $80^{\circ}$ to the sample surface with a nine-channel monolithic Ge fluorescence detector (E\&G Ortec Inc.) $)^{21}$ at $90^{\circ}$ to the beam in order to minimize the collected flux of backscattered X-rays. This results in a minimum path length in the fluid of $0.8 \mathrm{~mm}$, but sample roughness can increase this. The electrochemical cell was mounted so that the sample surface was in a vertical plane.

Data Processing. The XAS and ODXAS data were normalized and background corrected using a linear fit to the region between 150 and $30 \mathrm{eV}$ below the edge and normalized to a spline fitted between 10 and $500 \mathrm{eV}$ above the edge. Athena version 8.05 was used to process the data. ${ }^{22}$ In order to align the spectra from different experiments, the energy shift required to superimpose the edge regions from copper measurements made on each beam line/experiment was found, and the spectra were positioned with the $1 \mathrm{~s}-3 \mathrm{~d}$ edge resonance just below the ionization energy of 8.989 $\mathrm{keV}$. The same shift was then applied to other spectra from the same experiment. In cases where fitting was done, a simple oneparameter fit of a linear mixture of two relevant reference spectra was carried out after alignment of the data. The reference spectra were those obtained using XAS from the pure material.

\section{RESULTS AND DISCUSSION}

Figure 2 shows a logarithmic plot of XAS and ODXAS raw counts as a function of beam energy for a thin layer of cuprite on copper. Typically, over all the data presented in this paper, the ODXAS postedge signal was a factor of 3-5 lower than that in the XAS spectra, although in one case (nantokite on copper, discussed below) it was similar. This is entirely due to the

(12) Poolton, N. R. J.; Bøtter-Jensen, L.; Denby, P. M.; Nakamura, T.; Hamilton, B.; Pantos, E. Nucl. Instrum. Methods B 2004, 225, 590-598.

(13) Martínez-Criado, G.; Alen, B.; Homs, A.; Somogyi, A.; Miskys, C.; Susini, J.; Pereira-Lachataignerais, J.; Martínez-Pastor, J. Appl. Phys. Lett. 2006 89, 2219131-3.

(14) Sham, T. K.; Naftel, S. J.; Kim, P.-S. G.; Sammynaiken, R.; Tang, Y. H.; Coulthard, I.; Moewes, A.; Hu, Y.-F.; Lee, S. T. Phys. Rev. B 2004, 70 (3), 045313-045321. experimental setup since the measuring techniques and collection efficiencies are very different, and the input flux at ESRF (for the XAS) is 3-10 times greater than that available at SRS (for the ODXAS). Moreover, whereas the XAS signal rises typically more than 3 orders of magnitude across the edge, the ODXAS data sit on a high background due to nonresonant excitation processes and more than one optical emission mode. This limits the signal change at the edge to a factor of 3 at best and sometimes less than $50 \%$. The postedge data contain both the statistical fluctuation on the background level and that on the XAS itself, and this is made more evident (although not changed in absolute magnitude) by the mean background subtraction and normalization scheme normally used in XAS (i.e., the signal fluctuation is up to double that expected from Poisson statistics).

For this initial study, the unfiltered broadband optical radiation was used. It is intended to carry out a more detailed characterization of the optical emission spectra at a later date, to establish the optimum band for observing particular compounds, to reduce the background level where this is preferentially emitted in bands different from those carrying the ODXAS information, and to investigate the use of other fluorescence and phosphorescence channels which are also present. Since filtering to remove the background should be more efficient than simply acquiring more counts in both background and ODXAS channels we did not attempt to improve the ODXAS data by counting for the 3-10 times longer which would have been required. In any case, this would have placed the experiments outside a useful time resolution.

Powders. We first set out to see whether copper and its corrosion products could be uniquely identified from the ODXAS spectra using the pure reference samples. Since the detection mechanism differs from that of XAS, we also compare the ODXAS and XAS data from the same samples. The data for copper, cuprite, nantokite, and paratacamite are summarized in Figure 3.

Figure $3 \mathrm{a}$ shows the data obtained from the copper disk electrode. The ODXAS spectrum is noisier than the XAS in this and successive figures for the reasons described above. However, the spectra show similar features in both the XANES and EXAFS regions. The same is true for cuprite powder, shown in Figure $3 \mathrm{~b}$. The copper and cuprite spectra are easily distinguishable. The copper and cuprite XANES have the distinctive midedge peak due to the $1 \mathrm{~s}-3 \mathrm{~d}$ resonance, with single and double postedge features, respectively. The spectra for nantokite powder are shown in Figure 3c. While they are distinctive, they are also somewhat different between XAS and ODXAS with the ODXAS showing a broader spike on the edge and a differently structured postedge region. Moreover, there is no useful EXAFS information in the optically detected spectrum, a reproducible property of the unfiltered

(15) Kim, P.-S. G.; Hu, Y. F.; Brandys, M. C.; Burchell, T. J.; Puddephatt, R. J.; Sham, T. K. Inorg. Chem. 2007, 46 (3), 949-957.

(16) Scott, D. A. Copper and Bronze in Art: Corrosion, Colorants, Conservation; The Getty Conservation Institute: Los Angeles, 2002.

(17) Lamy, C. Stabilisation d'Objets Archéologiques Chlorurés en Alliage Cuivreux, Report ARC'Antique, Nantes, France, 1997.

(18) Pollard, A. M.; Thomas, R. G.; Williams, P. A. Minerol. Mag. 1989, 53, 557-563.

(19) Boydag, F. S.; Lenger Ozcanl, Y.; Alekperov, V. A. J. Appl. Spectrosc. 2007, 74, 752-759.

(20) Quinn, F.; Poolton, N. R. J.; Malins, A.; Pantos, E.; erson, C.; Denby, P.; Dhanak, V.; Miller, G. J. Synchrotron Radiat. 2003, 10, 461-466.

(21) Derbyshire, G.; Cheung, K. C.; Sangsingkeow, P.; Hasnain, S. S. J. Synchrotron Radiat. 1999, 6, 62-63.

(22) Ravel, B.; Newville, M. J. Synchrotron Radiat. 2005, 12, 537-541. 


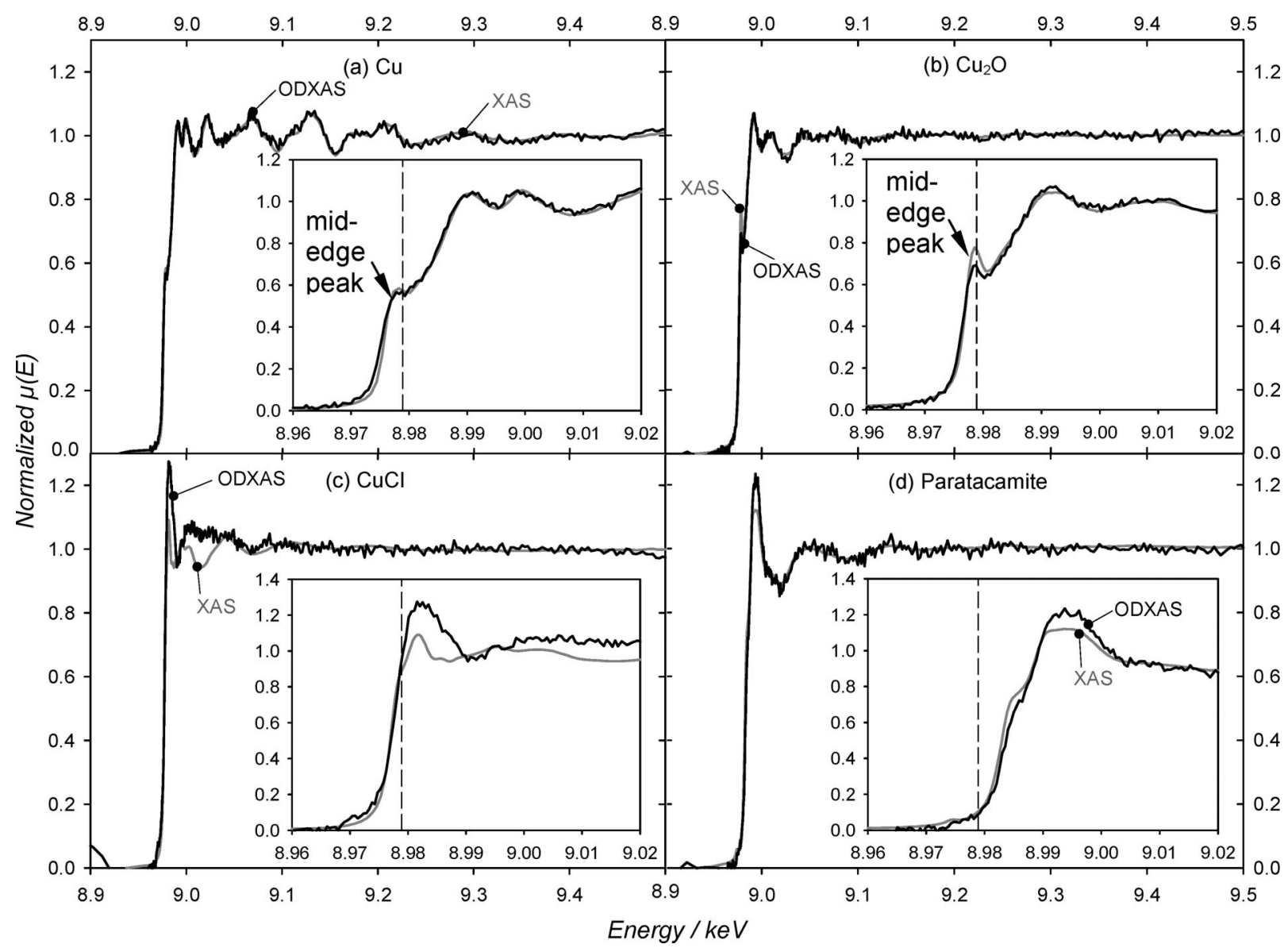

Figure 3. XAS and ODXAS spectra of copper (a) and the three reference powders: cuprite (b), nantokite (c), and atacamite/paratacamite (d). The inset shows the region of the edge in more detail with the dashed line at the copper ionization energy of $8.979 \mathrm{keV}$.

optical data for all ODXAS spectra from nantokite powder. Taken together, these two features of the spectrum suggest that a photonic absorption mechanism is occurring in the powder in a way similar to self-absorption for X-rays in a thick sample. In contrast, we show later that ODXAS from thin nantokite layers on copper are very similar to XAS from powder samples, and do contain identical EXAFS modulation. Paratacamite (Figure 3d) has very similar spectra in both XAS and ODXAS and is also distinct from the other reference spectra. As one might expect, paratacamite and atacamite (the latter not shown in Figure 3) also present highly similar ODXAS spectra due to very similar octahedral coordination of the copper in the solid. ${ }^{23}$ In addition, the production of paratacamite also produces significant amounts of atacamite, and it is likely that the converse is true. There is no observable difference in the spectra from atacamite and paratacamite prepared by the different methods.

Dry Corrosion Layers. The focus of our corrosion studies is the real-time monitoring of conservation treatments on corroded copper and copper alloy substrates. Not only may the underlying copper be visible in spectra of such samples but the corrosion layer may have structural (and maybe even chemical) differences from the pure reference powder.

Spectra from the cuprite reference, copper and from a cuprite layer on the surface of a copper working electrode, taken with

(23) Wells, A. F. Acta Crystallogr. 1949, 2, 175-180.

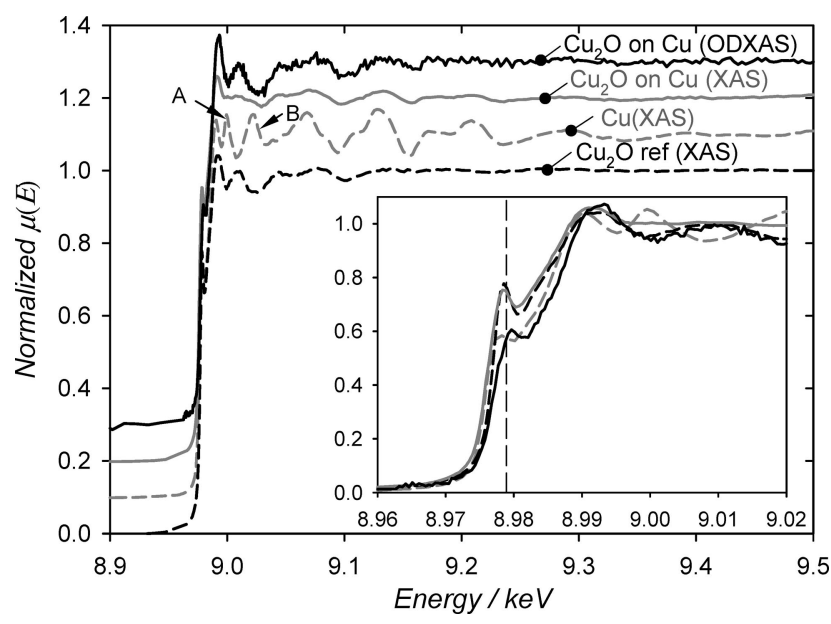

Figure 4. XAS and ODXAS spectra of a dry cuprite layer on a copper coupon compared with XAS of a cuprite powder reference and a clean copper coupon. The enhanced cuprite spectral content of the layer ODXAS in comparison with the XAS is obvious, the latter comprising a superposition of copper and cuprite spectra. In the main figure, spectra have been offset by 0.1 in the vertical direction. The inset shows the edge detail with no offset.

XAS and ODXAS are compared in Figure 4. The spectra in the main figure are spaced vertically by adding multiples of 0.1 to the intensity, but those in the inset are as normalized. The same presentation is used for the rest of the figures in the paper. 


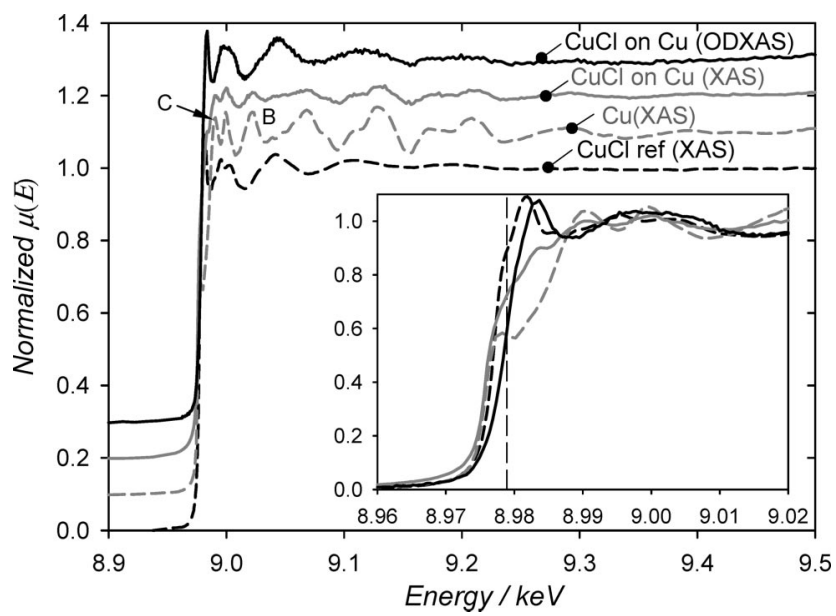

Figure 5. XAS and ODXAS spectra of a dry nantokite layer on copper compared with a $\mathrm{CuCl}$ reference powder and copper metal. The surface specificity of the ODXAS measurement is very apparent in the similarity of the nantokite on copper spectrum and that of the pure material (black continuous and dashed lines, respectively) and the absence of the copper-related modulation at (C) and (B) (arrows), which is prominent in the XAS spectrum for the corrosion layer on copper. The relative freedom from noise on the ODXAS arises from the large optical fluorescence from $\mathrm{CuCl}$ combined with a signal change at the edge greater than a factor of 2 .

The XAS spectrum is clearly a superposition of the spectra of the cuprite layer and the underlying copper, and the copper contribution is significant because the range of the $K \alpha$ fluorescence exceeds the thickness of the cuprite. Fitting shows the relative contributions to be $45 \% \mathrm{Cu}$ and $55 \% \mathrm{Cu}_{2} \mathrm{O}$ with a misfit $\left(\chi^{2}\right)$ of 0.013 . The optical transparency of cuprite depends on its morphology and many other factors, but in general, it has minimal transmittance from UV to $550 \mathrm{~nm}$, which then increases into the IR. $^{24}$ ODXAS might therefore produce a spectrum much more closely resembling that of the cuprite powder, provided that significant optical fluorescence occurs above $550 \mathrm{~nm}$. This essential surface specificity is confirmed in Figure 4 through the close similarity between the ODXAS spectrum (black solid line) and the reference spectrum from $\mathrm{Cu}_{2} \mathrm{O}$ powder (black dashed line) and by noting that the copper modulations at (A) and (B) suppress the modulation in the XAS but not the ODXAS spectrum. In this case, the best fit was obtained with $100 \% \mathrm{Cu}_{2} \mathrm{O}$, with a larger misfit $(0.03)$ because of the fluctuation in the data.

The same relationship between the powder and layer spectra can be seen for nantokite in Figure 5. Indeed, the ODXAS spectrum for the nantokite layer is very similar to that for XAS of the powder (best fit $98 \% \mathrm{CuCl}, 2 \% \mathrm{Cu}, \chi^{2}=0.12$ ) with strong EXAFS modulation (comparing the black solid line and the black dashed line as before). Note also that the copper-related features at (B) and (C) in the figure also appear prominently in the XAS for nantokite on copper (overall best fitted by $74 \% \mathrm{Cu}$ plus $26 \%$ $\left.\mathrm{CuCl}, \chi^{2}=0.08\right)$ but not in the ODXAS. This contrasts with the data for nantokite powder, which consistently gave a different and noisy spectrum (as mentioned above).

The XAS spectrum for an atacamite layer on a copper substrate is dominated by the atacamite spectrum, with no measurable contribution from the underlying copper because of the thickness

(24) Liu, Y. L.; Liu, Y. C.; Mu, R.; Yang, H.; Shao, C. L.; Zhang, J. Y.; Lu, Y. M.; Shen, D. Z.; Fan, X. W. Semicond. Sci. Technol. 2005, 20, 44-49.

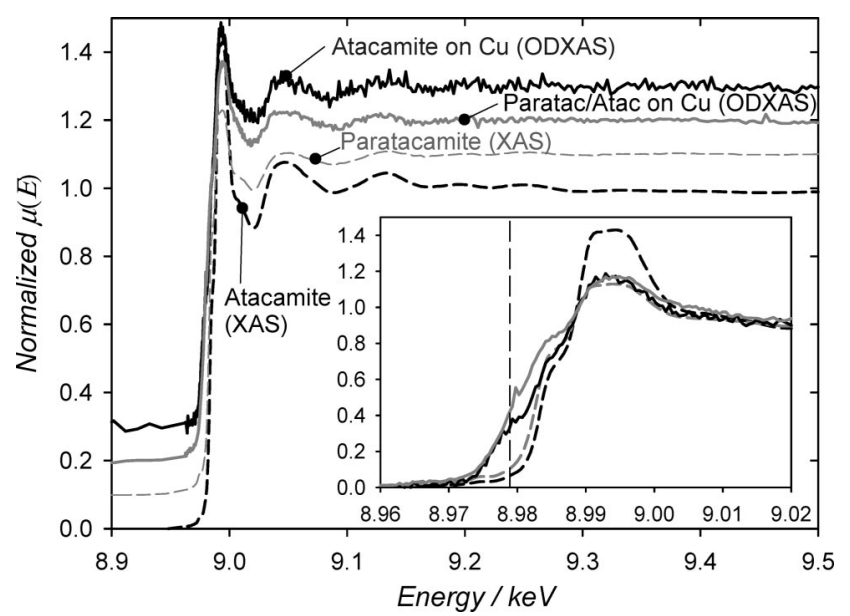

Figure 6. ODXAS of atacamite and mixed atacamite/paratacamite layers on copper compared with XAS of powder references.

of the corrosion. This is shown in Figure 6. ODXAS again only measures the spectrum of the atacamite (or paratacamite) layer, but the edge slope is rather lower than for XAS, suggesting some visible photon excitation from processes due to copper excitation rather than ionization.

LDPE Window, Electrolyte, and Noise Levels. When ODXAS measurements are made on a sample in the eCell, the X-ray beam must pass through both the LDPE cell window and a layer of electrolyte, in addition to the electrode surface. Photons in the near-UV to near-IR band must also pass through the electrolyte and window. We therefore made comparative measurements with and without various window/fluid layer combinations to establish their contribution to the electrode measurements.

The LDPE window alone does not affect the observed spectrum in any measurable way, so its presence in spectroelectrochemical procedures should not affect the results of ODXAS analyses. (Although our measurements show that the window itself absorbs copper compounds from the electrolyte and must be renewed for each experiment. Similar absorption of chlorides and attachment of oxide particles is observed for Kapton and PET windows as well. ${ }^{5}$ )

The presence of the solution apparently makes the signal considerably noisier, but the cuprite spectrum is still discernible, although it would not be useful for structural determination. In general, the presence of a fluid layer this thick reduces the relative height of the edge because the background is increased, thus making the noise more significant after normalization. XAS and ODXAS data are also affected by absorption and scattering of X-rays on the input path and XAS is attenuated on the escape path as well. However, the presence of sodium sesquicarbonate over the sample always increases the relative background level in ODXAS, showing that extra optical emission is coming from the sodium salt solution.

Electrodes in Sodium Sesquicarbonate Solution. The ODXAS spectrum of a copper working electrode, both dry, and immersed in sodium sesquicarbonate solution with the cell window in place, are depicted in Figure 7. The solution makes the normalized copper spectrum noisier, as expected in view of the previous result. Conversely, the XAS spectra of cuprite on a copper substrate both dry and immersed are identical (In both cases, the spectrum is dominated by the copper (cf. Figure 2(a)) but the midedge peak is a little more prominent because of the cuprite.). 


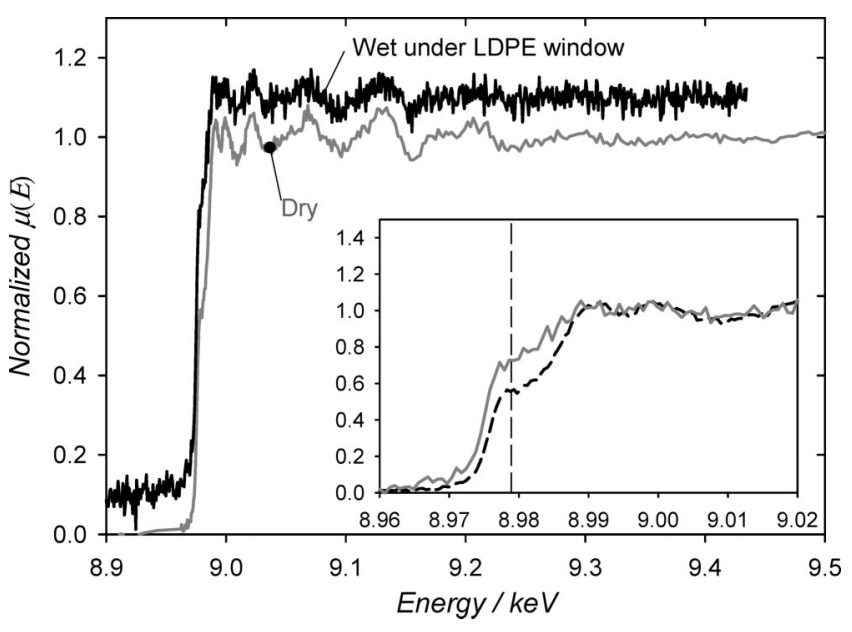

Figure 7. ODXAS spectrum of copper in contact with a $1 \%(\mathrm{w} / \mathrm{v})$ sodium sesquicarbonate solution and behind a 10- $\mu \mathrm{m}$-thick LDPE window. The fluid pocket between the surface and the window is $\sim 125$ $\mu \mathrm{m}$ thick. The increased noise compared to the dry spectrum is due to a drop of a factor of 3.5 in signal intensity.

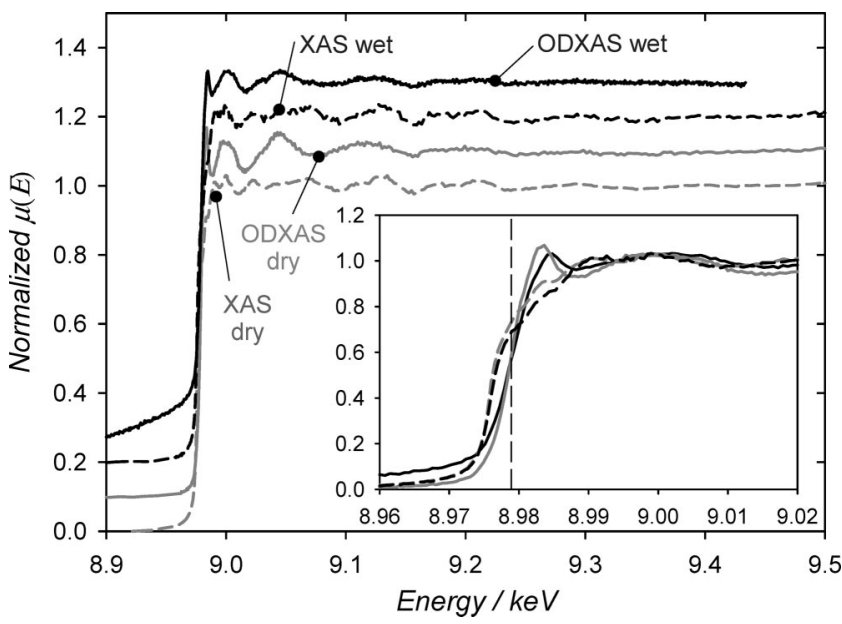

Figure 8. Comparison of dry XAS and ODXAS spectra of a nantokite layer on a copper substrate with the same layer in contact with a $1 \%$ $(\mathrm{w} / \mathrm{v})$ sodium sesquicarbonate solution retained by a $10-\mu \mathrm{m}$-thick LDPE window.

XAS and ODXAS spectra of nantokite on a copper substrate both dry and immersed are shown in Figure 8. As with the cuprite sample above, the XAS spectrum is unmodified by the solution, but relatively uninformative, dominated as it is by copper-related features. In this case, the ODXAS data are also relatively noise-free, which we found to be typical of nonpowder nantokite scans, and the high surface specificity is clearly preserved. Examination of the raw data for the ODXAS showed the count rate to be $5-10$ times greater for the same input flux and other conditions, and these spectra also have a change in signal of a factor of $2-3$ at the edge-clearly the conversion to visible photons carrying the absorption spectrum is very efficient in this material.

In paratacamite and atacamite (thick layers), the wetting process reduces the magnitude of the postedge oscillations in ODXAS, but not in XAS as shown in Figure 9. Here, the ODXAS is specific to the hydroxychloride surface, whereas the XAS is more characteristic of the bulk corrosion layer. Immersion in sesquicarbonate modifies the surface of the hydroxychloride layer quite rapidly. This is supported

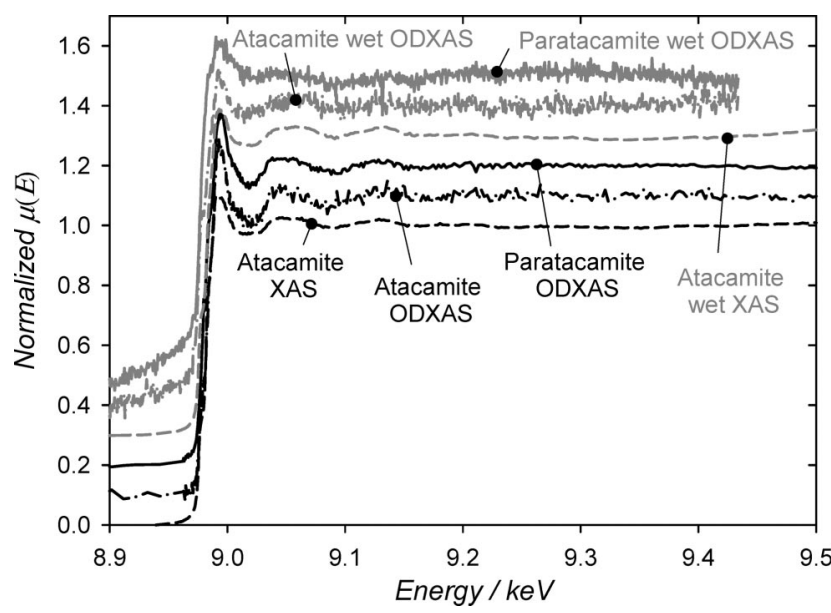

Figure 9. Comparison of XAS and ODXAS from dry atacamite and atacamite/paratacamite layers on copper coupons with similar layers in contact with a $1 \%(\mathrm{w} / \mathrm{v})$ sodium sesquicarbonate solution retained by a $10-\mu \mathrm{m}$-thick LDPE window.

by XAS, XRD, and RBS data to be published elsewhere, ${ }^{5}$ which show that these synthetic layers have a rather soluble but octahedrally coordinated "skin" overlying the bulk material.

\section{CONCLUSIONS}

ODXAS has been shown to be a very promising technique for the future study of corrosion both in air and in a liquid environment. Copper, copper(I) oxide (cuprite), copper(I) chloride (nantokite), and copper(II) trihydroxychloride all have distinct and recognizable spectra, and the optically detected spectra are consistent with the conventionally detected XAS spectra for bulk powders, except in the case of nantokite, where the thin-layer ODXAS has nearly identical structure to the bulk powder XAS.

We show that the technique is significantly more surface specific than conventional XAS, even in the unfiltered broadband data presented here. Whereas the copper substrate signal dominates in the case of XAS of thin (i.e., $<1.5 \mu \mathrm{m}$ ) nantokite and cuprite layers (with up to $75 \%$ of copper in the fit), the compound signal dominates in the ODXAS $(>97 \%)$. This makes ODXAS an attractive prospect for the observation of corrosion layers in situ, especially where these are thin. In addition, comparison between ODXAS and XAS data may give an indication of stratigraphy within a corrosion layer.

The presence of the LDPE cell window on its own does not have a measurable effect on either the XAS or ODXAS spectrum. However, when this is combined with a $\sim 125-\mu$ m-thick electrolyte pocket, the background in ODXAS is increased and the proportional step at the edge is reduced. This leads to a significant increase in the relative statistical fluctuation in the normalized data because the absolute statistical fluctuation of the raw count is retained. Nevertheless, where the postedge to pre-edge signal ratio exceeds a factor of 2 (as is the case for nantokite layers reported here), background-subtracted and normalized ODXAS spectra have acceptable noise levels. We are constructing a new instrument with 10 times the collection efficiency of that used in these experiments. This incorporates band-pass filtering to alleviate the statistical effects of postacquisition background removal where the bands carrying the XANES and EXAFS data are distinct from those of the background. 


\section{ACKNOWLEDGMENT}

The authors gratefully acknowledge the following for their help: Derrick Richards, Pieter van Hoe, and Adrian Lovejoy (cell construction); Ingrid Oloff, Hans Buschop, Karen Leyssens, and Bart Schotte (help with the analyses). The eCell was developed using private funds from EVA Surface Analysis (UK) and lately from the Paul Instrument fund. The work was partly supported by SRS Daresbury and the Fund for Scientific Research-Flanders.

Received for review May 1, 2008. Accepted September 1, 2008.

AC800895N 